PROCEEDINGS OF THE

AMERICAN MATHEMATICAL SOCIETY

Volume 127, Number 9, Pages 2611-2618

S 0002-9939(99)04824-8

Article electronically published on April 23, 1999

\title{
ON THE NONRATIONALITY OF RIGID LIE ALGEBRAS
}

\author{
J. M. ANCOCHEA BERMUDEZ AND M. GOZE
}

(Communicated by Roe Goodman)

\begin{abstract}
In his thesis, Carles made the following conjecture: Every rigid Lie algebra is defined on the field $Q$. This was quite an interesting question because a positive answer would give a nice explanation of the fact that simple Lie algebras are defined over $Q$. The goal of this note is to provide a large number of examples of rigid but nonrational and nonreal Lie algebras.
\end{abstract}

\section{Rigid Lie ALGEBRAS} $[2])$.

In this section, we briefly recall some properties of rigid Lie algebras (see [1],

1.1 Definition. A Lie algebra law on $\mathbb{C}^{n}$ is a bilinear mapping

$$
\mu: \mathbb{C}^{n} \times \mathbb{C}^{n} \longrightarrow \mathbb{C}^{n}
$$

satisfying

$$
\left\{\begin{array}{l}
\mu(X, Y)=-\mu(Y, X) \quad \forall X, Y \in \mathbb{C}^{n}, \\
\mu(\mu(X, Y), Z)+\mu(\mu(Y, Z), X)+\mu(\mu(Z, X), Y)=0 \quad \forall X, Y, Z \in \mathbb{C}^{n} .
\end{array}\right.
$$

The last equations are defined by the Jacobi conditions. If we fix a basis $\left\{e_{i}\right\}$ of $\mathbb{C}^{n}$, we can identify the law $\mu$ with its structural constants $C_{i j}^{k}$ defined by $\mu\left(e_{i}, e_{j}\right)=$ $\sum_{k=1}^{n} C_{i j}^{k} e_{k}$. The conditions (1) are translated in terms of $C_{i j}^{k}$ by

$$
\left\{\begin{array}{l}
C_{i j}^{k}=-C_{j i}^{k}, \\
\sum_{l=1}^{n} C_{i j}^{l} C_{k l}^{s}+C_{j k}^{l} C_{i l}^{s}+C_{k i}^{l} C_{j l}^{s}=0,1 \leq i<j<k \leq n, 1 \leq s \leq n .
\end{array}\right.
$$

Let $\mathcal{L}^{n}$ be the algebraic variety parametrized by the $\left(C_{i j}^{k}\right)$ and defined by the polynomial equations (2). We identify a law $\mu$ with the corresponding point in $\mathcal{L}^{n}$ and a Lie algebra $\mathfrak{g}$ with its law $\mu$. The linear group $G l(n, \mathbb{C})$ acts on $\mathcal{L}^{n}$ by

$$
\begin{gathered}
G l(n, \mathbb{C}) \times \mathcal{L}^{n} \longrightarrow \mathcal{L}^{n}, \\
f, \quad \mu \longrightarrow f^{-1} \mu(f, f)
\end{gathered}
$$

with $f^{-1} \mu(f, f)(X, Y)=f^{-1}(\mu(f(X), f(Y))$. The orbit $\Theta(\mu)$ of $\mu$, which corresponds to the class of laws which are isomorphic to $\mu$, is a smooth subvariety of $\mathcal{L}^{n}$.

Received by the editors April 19, 1996 and, in revised form, December 1, 1997.

1991 Mathematics Subject Classification. Primary 17Bxx.

Key words and phrases. Rigid Lie algebras. 
Definition 1. The Lie algebra law $\mu$ is rigid if its orbit $\Theta(\mu)$ is an open set of $\mathcal{L}^{n}$ (endowed with the Zariski topology).

If $\mu$ is rigid, the Zariski closure $\overline{\Theta(\mu)}$ of the orbit is an irreducible algebraic component of $\mathcal{L}^{n}$. As every algebraic variety is a finite union of irreducible algebraic components, we have only a finite number of isomorphic classes of rigid algebra laws.

The notion of rigidity can be translated in infinitesimal terms. Suppose that $\mu_{0}$ is a standard given law in $\mathcal{L}^{n}$. A perturbation of $\mu_{0}$ is a law $\mu \in \mathcal{L}^{n}$ whose structure constants with respect to a standard basis of $\mathbb{C}^{n}$ are infinitely close to the constants of $\mu_{0}$. The law $\mu_{0}$ is rigid if and only if every perturbation of $\mu_{0}$ is in $\Theta\left(\mu_{0}\right)$. This point of view is very practical for proving the rigidity of a given law: we consider a general infinitesimal perturbation and we solve the infinitesimal polynomial system given by the nonstandard structure constants. For example, Theorem 2 is proved in this way. This approach is developed in [1] and [7].

\subsection{Structure of rigid Lie algebras.}

Theorem 1 ([4]). A rigid complex Lie algebra $\mathfrak{g}$ is decomposable, that is, it can be written $\mathfrak{g}=\mathfrak{s} \oplus \mathfrak{u} \oplus \mathfrak{n}$ where $\mathfrak{s}$ is a Levi semisimple subalgebra, $\mathfrak{u}$ a subalgebra whose elements are ad($(\mathfrak{g})$-semisimple, and $\mathfrak{n}$ is the nilradical of $\mathfrak{g}$.

Now suppose that $\mathfrak{g}$ is a rigid solvable Lie algebra. Then its decomposition is $\mathfrak{g}=\mathfrak{u} \oplus \mathfrak{n}$. A vector $X \in \mathfrak{u}$ is called regular if $\operatorname{dim} \operatorname{Ker}$ adX is minimal among all the vectors of $\mathfrak{u}$. We fix a regular vector $X$, and we consider a basis $\left\{e_{i}\right\}$ of eigenvectors of $a d X$ with $e_{1}=X$. The linear system

$$
S(X)=\left\{C_{i j}^{k}\left(x_{i}+x_{j}-x_{k}\right)=0 \quad 2 \leq i<j<k \leq n\right\}
$$

has been introduced in [2]. Its role is summarized in the following property:

Theorem 2. If $\mathfrak{g}$ is rigid, the rank of $S(X)$ satisfies $\operatorname{rg}(S(X))=\operatorname{dim} \mathfrak{n}-1$.

Consequence 1. If $\mathfrak{g}=\mathfrak{u} \oplus \mathfrak{n}$ is a rigid solvable Lie algebra, then $\mathfrak{u}$ is a maximal subalgebra whose elements are ad $\mathfrak{g}$-semisimple.

Then $\mathfrak{u}$ corresponds to an exterior torus of derivations of the nilradical $\mathfrak{n}$ in a Malcev sense. For this reason we will denote this subalgebra by $\mathfrak{t}$ (which henceforth will be maximal).

Consequence 2. There is a basis $\left(X_{1}, X_{2}, \ldots, X_{n}\right)$ for $\mathfrak{t}$ such that the eigenvalues of the semisimple operators ad $X_{i}$ are integers.

\section{ON THE NONRATIONALITY OF RIGID LIE ALGEBRAS}

A Lie algebra is called rational if it is defined over the field $\mathbb{Q}$. The conjecture that rigid Lie algebras are rational is based on the following examples:

1. Every semisimple complex Lie algebra is rigid. Recall that these algebras are defined on $\mathbb{Q}$.

2. Every solvable complex rigid Lie algebra of dimension less than 8 is rational. We note that the classification of rigid Lie algebras is known only up to dimension 8 [1].

3. There is a regular vector $X$ such that the eigenvalues of adX are integers.

Unfortunately, this conjecture is false. The aim of this paper is to construct some general examples of nonrational rigid Lie algebras. These examples are found by studying the rigid solvable Lie algebras whose nilradicals have maximal nilindex. 
Theorem 3. The following nonrational Lie algebras of dimension 13 are rigid:

$$
\left\{\begin{array}{l}
{\left[X, Y_{i}\right]=i Y_{i}, i=1, \cdots, 11,\left[X, Y_{12}\right]=13 Y_{12}} \\
{\left[Y_{1}, Y_{i}\right]=Y_{i+1}, i=2, \cdots, 10} \\
{\left[Y_{i}, Y_{13-i}\right]=(-1)^{i} Y_{12}, \quad 2 \leq i \leq 6} \\
{\left[Y_{i}, Y_{j}\right]=a_{i j} Y_{i+j}}
\end{array}\right.
$$

with the coefficients $a_{i j}$ satisfying $a_{i j}=a_{i j+1}+a_{i+1 j}$ being linear combinations of $\alpha_{1}=a_{23}, \alpha_{2}=a_{34}, \alpha_{3}=a_{45}, \alpha_{4}=a_{56}$ with $\alpha_{2}=\frac{1+\sqrt{10}}{3}$, or $\alpha_{2}=\frac{1-\sqrt{10}}{3}$, or $\alpha_{2}=\frac{2+i \sqrt{2}}{3}$, or $\alpha_{2}=\frac{2-i \sqrt{2}}{3}$.

We will see that the two last rigid Lie algebras are nonreal. The proof is a direct consequence of Proposition 3.

\section{Filiform Lie algebras With a nONnUll Rank [5]}

An $n$-dimensional nilpotent Lie algebra $\mathfrak{n}$ is filiform if its nilindex

$$
i(\mathfrak{n})=\operatorname{Max}\left\{p \text { such that } \exists X \in \mathfrak{n} \text { with }(\operatorname{adX})^{p} \neq 0\right\}
$$

is equal to $n-1$. Another interesting notion for nilpotent Lie algebras is the notion of rank. The rank $r(\mathfrak{n})$ of $\mathfrak{n}$ is the dimension of a maximal exterior torus of derivations (recall that all maximal tori have the same dimension). In [5], we have determined all the filiform Lie algebras having a nonnull rank (A different approach of this problem can be found in [8]). We sum up these results.

The rank of a filiform Lie algebra is less than or equal to 2.

Every $n$-dimensional filiform Lie algebra of rank 2 is isomorphic to

$$
\begin{gathered}
i) \mathfrak{n}=L_{n} \\
{\left[Y_{1}, Y_{i}\right]=Y_{i+1} \quad 2 \leq i \leq n-1,} \\
{\left[Y_{i}, Y_{j}\right]=0 \quad 2 \leq i, j \leq n .} \\
i i) \mathfrak{n}=Q_{n}, \quad n=2 m, \\
{\left[Y_{1}, Y_{i}\right]=Y_{i+1}, \quad 2 \leq i \leq n-2,} \\
{\left[Y_{i}, Y_{n-i+1}\right]=(-1)^{i+1} Y_{n}, \quad 2 \leq i \leq n-1 .}
\end{gathered}
$$

Every $n$-dimensional filiform Lie algebra of rank 1 is isomorphic to

$$
\begin{gathered}
\text { iii }) \mathfrak{n}=A_{n}^{k}\left(\alpha_{1}, \cdots, \alpha_{t}\right), 2 \leq k, \quad t=\left[\frac{n-k-1}{2}\right], \\
{\left[Y_{1}, Y_{i}\right]=Y_{i+1} \quad 2 \leq i \leq n-1,} \\
{\left[Y_{i}, Y_{i+1}\right]=\alpha_{i-1} Y_{2 i+k-1}, \quad 2 \leq i \leq t+1,} \\
{\left[Y_{i}, Y_{j}\right]=a_{i j} Y_{i+j+k-2}, 2 \leq i<j \text { and } i+j+k-2 \leq n .}
\end{gathered}
$$




$$
\begin{gathered}
i v) \mathfrak{n}=B_{n}^{k}\left(\alpha_{1}, \cdots, \alpha_{t}\right), n=2 m, 2 \leq k \leq n-3, t=\left[\frac{n-k}{2}\right] \\
{\left[Y_{1}, Y_{i}\right]=Y_{i+1} \quad 2 \leq i \leq n-2,} \\
{\left[Y_{i}, Y_{n-i+1}\right]=(-1)^{i+1} Y_{n} \quad 2 \leq i \leq n-1,} \\
{\left[Y_{i}, Y_{i+1}\right]=\alpha_{i-1} Y_{2 i+k-1}, \quad i=2, \cdots,\left[\frac{n-k-1}{2}\right],} \\
{\left[Y_{i}, Y_{j}\right]=a_{i j} Y_{i+j+k-2}, \quad 2 \leq i, j \leq n-2 \text { et } i+j+k-2 \leq n-1, j \neq i+1} \\
{\left[Y_{i}, Y_{n-i+1}\right]=(-1)^{i-1} Y_{n} \quad 2 \leq i \leq m+1,} \\
{\left[Y_{i}, Y_{n-i-2 k+1}\right]=(-1)^{i+1} \alpha_{k} Y_{n}, 2 \leq i \leq n-2-2 k \text { et } 1 \leq k \leq m-1}
\end{gathered}
$$

Where $\left(\alpha_{1}, \cdots, \alpha_{t}\right)$ are parameters satisfying polynomial relations corresponding to the Jacobi conditions.

Remark. In [3] we have studied the rigid Lie algebras whose nilradical is isomorphic to $A_{n}^{k}$ and we have obtained a nonrational example. The next section is devoted to the study of rigid Lie algebras whose nilradical is isomorphic to $B_{n}^{k}$. Note that the case $\mathfrak{n}=C_{n}$ is excluded because it is impossible to have rigid algebras with such nilradical [3].

\section{Rigid Lie algebras Whose nilradical is $B_{n}^{k}$}

Let $\mathfrak{g}=\mathfrak{t} \oplus \mathfrak{n}$ be a rigid Lie algebra whose nilradical $\mathfrak{n}$ is isomorphic to the filiform algebra $B_{n}^{k}(n=2 m)$. In this case $\operatorname{dim} \mathbf{t}=1$, and the eigenvalues of $a d X, X \in \mathfrak{t}-\{0\}$, are $k, k+1, \cdots, k+2 m-3,2 k+2 m-3$.

Proposition 1. Let $\mathfrak{g}=\mathfrak{t} \oplus \mathfrak{n}$ be a decomposable solvable Lie algebra with $\mathfrak{n}=B_{n}^{k}$, and $n=2 m$.

1. If $k \geq 2 m-4$, then $\mathfrak{g}$ is not rigid.

2. If $k=2 m-4$, then $\mathfrak{g}$ is isomorphic to

$$
\left\{\begin{array}{l}
{\left[X, Y_{1}\right]=Y_{1} ;\left[X, Y_{i}\right]=(k+i-2) Y_{i}, i=2, \cdots, n-1 ;\left[X, Y_{n}\right]=(3 k+1) Y_{n},} \\
{\left[Y_{1}, Y_{i}\right]=Y_{i+1}, i=2, \cdots, n-2 ;\left[Y_{i}, Y_{n-i+1}\right]=(-1)^{i} Y_{n}, i=2, \cdots, n-2,} \\
{\left[Y_{2}, Y_{3}\right]=Y_{n-1},}
\end{array}\right.
$$

which is a rational rigid Lie algebra.

3. If $k=2 m-5$, then $\mathfrak{g}$ is isomorphic to

$$
\left\{\begin{array}{l}
{\left[X, Y_{1}\right]=Y_{1},\left[X, Y_{i}\right]=(k+i-2) Y_{i} i=2, \cdots, n-1,\left[X, Y_{n}\right]=(3 k+2) Y_{n}} \\
{\left[Y_{1}, Y_{i}\right]=Y_{i+1} i=2, \cdots, n-2\left[Y_{2}, Y_{3}\right]=Y_{n-2},\left[Y_{2}, Y_{4}\right]=Y_{n-1}} \\
{\left[Y_{i}, Y_{n-i+1}\right]=(-1)^{i} Y_{n}}
\end{array}\right.
$$

which is rigid and rational. 
4. If $k=2 m-6, \mathfrak{g}$ is isomorphic to

$$
\left\{\begin{array}{l}
{\left[X, Y_{1}\right]=Y_{1} ;\left[X, Y_{i}\right]=(k+i-2) Y_{i}, i=2, \cdots, n-1 ;\left[X, Y_{n}\right]=(3 k+3) Y_{n},} \\
{\left[Y_{1}, Y_{i}\right]=Y_{i+1}, i=2, \cdots, n-2 ;\left[Y_{i}, Y_{n-i+1}\right]=(-1)^{i} Y_{n}, i=2, \cdots, n-2,} \\
{\left[Y_{2}, Y_{3}\right]=Y_{n-3} ;\left[Y_{2}, Y_{4}\right]=Y_{n-2} ;\left[Y_{2}, Y_{5}\right]=9 Y_{n-1} ;\left[Y_{3}, Y_{4}\right]=2 Y_{n-1},}
\end{array}\right.
$$

which is rigid and rational

5. If $k=2 m-7$, then $\mathfrak{g}$ is not rigid.

Proof. If $k \geq 2 m-4$, the nilradical $\mathfrak{n}$ is isomorphic to $Q_{n}$. From Theorem $2, \mathfrak{g}$ is not rigid.

Suppose $k=2 m-4$. Let $\mu_{0}$ be the law given in this proposition. Consider a perturbation $\mu$ of $\mu_{0}$. The adjoint operator $a d_{\mu} X$ is a perturbation of the standard operator $a d_{\mu_{0}} X$. But this last operator is diagonalizable and its eigenvalues are $0,1, k+i-2 \quad i=2, \cdots, n-1$ and $3 k+1$. So $a d_{\mu} X$ is also semisimple because its eigenvalues $0, \lambda_{i}$ satisfy $\lambda_{1} \simeq 1, \lambda_{i} \simeq k+i-2, \lambda_{n} \simeq 3 k+1$ and then $\lambda_{i} \neq \lambda_{j}$ for $i \neq j$ (the symbol $\simeq$ corresponds to infinitely close). Recall the following results of perturbation of linear operators: If $F_{\lambda}$ is the eigenspace associated to the eigenvalue $\lambda$ of the perturbed operator $a d_{\mu} X$, then the standard part (the shadow) of $F_{\lambda}$ is the eigenspace of the operator $a d_{\mu_{0}} X$ corresponding to the eigenvalue $\lambda_{0}$ satisfying $\lambda \bumpeq \lambda_{0}$. These two spaces have the same dimension. From this, we can find a basis $U_{1}, U_{2}, \cdots, U_{n}, X$ such that

$$
\begin{gathered}
U_{i} \simeq Y_{i} \quad i=1, \cdots, n, \\
\operatorname{ad}_{\mu} X\left(U_{i}\right)=\lambda_{i} U_{i} .
\end{gathered}
$$

As $\mu\left(U_{i}, U_{j}\right) \simeq \mu_{0}\left(Y_{i}, Y_{j}\right)$, and from the Jacobi identities, we have $\mu\left(U_{i}, U_{j}\right)=$ $a_{i j} U_{i+j}$. The eigenvalues $\lambda_{1}, \cdots, \lambda_{n}$ satisfy the system $S(X)$ (see section 1.2) relative to $\mu_{0}$. Thus, we have:

$$
\begin{gathered}
\lambda_{i}=(k+i-2) \lambda_{1}, i=2, \cdots, n-1, \\
\lambda_{n} \simeq(3 k+1) \lambda_{1} .
\end{gathered}
$$

Taking $Z=\frac{X}{\lambda_{1}}, Z_{1}=U_{1}, \quad Z_{2}=a U_{2}, \quad Z_{i}=a a_{12} a_{13} \cdots a_{1 i-1} U_{i}, \quad i=3, \cdots, n-1$ and $Z_{n}=a^{2} a_{12} a_{13} \cdots a_{1 n-2} a_{2 n-1} U_{n}$ with $a=\frac{a_{13} \cdots a_{1 n-2}}{a_{23}}$, we see that the structure constants of $\mu$ with respect the basis $Z_{1}, \cdots, Z_{n}, Z$ are the same as the structure constants of $\mu_{0}$ with respect to the basis $Y_{1}, \cdots, Y_{n}, X$. Thus, the laws $\mu$ and $\mu_{0}$ are isomorphic and $\mu_{0}$ is rigid.

The cases $k=2 m-5$ and $k=2 m-6$ are similar.

Suppose $k=2 m-7$. We have the following Lie algebras:

$$
\left\{\begin{array}{l}
{\left[X, Y_{1}\right]=Y_{1} ;\left[X, Y_{i}\right]=(k+i-2) Y_{i}, i=2, \cdots, n-1 ;\left[X, Y_{n}\right]=(3 k+4) Y_{n},} \\
{\left[Y_{i}, Y_{n-i+1}\right]=(-1)^{i} Y_{n}, i=2, \cdots, n-2,} \\
{\left[Y_{2}, Y_{3}\right]=\alpha_{1} Y_{n-4} ;\left[Y_{2}, Y_{4}\right]=\alpha_{1} Y_{n-3} ;} \\
{\left[Y_{2}, Y_{5}\right]=\left(\alpha_{1}-\alpha_{2}\right) Y_{n-2} ;\left[Y_{2}, Y_{6}\right]=\left(\alpha_{1}-2 \alpha_{2}\right) Y_{n-1},} \\
{\left[Y_{3}, Y_{4}\right]=\alpha_{2} Y_{n-2} ;\left[Y_{3}, Y_{5}\right]=\alpha_{2} Y_{n-1} .}
\end{array}\right.
$$

The parameters $\alpha_{1}$ and $\alpha_{2}$ are free (all the Jacobi conditions are satisfied). These Lie algebras are nonrigid. 
Other fundamental cases correspond to the small values of $k$. We begin, of course, with $k=2$ (if $k=1$, then $\mathfrak{n}=Q_{n}$ and $\operatorname{dim} \mathfrak{t}=2$ ). If $\operatorname{dim} \mathfrak{g}=7$ or 9 , then $k=2 m-4$ or $2 m-6$. Theses algebras have been studied above.

Proposition 2. Let $\mathfrak{g}=\mathfrak{t} \oplus \mathfrak{n}$ be a decomposable solvable Lie algebra with $\mathfrak{n}=B_{n}^{2}$, and $n=2 m$.

1. If $\operatorname{dim} \mathfrak{g}=7$ or 9 , then $\mathfrak{g}$ is rigid and rational.

2. If dim $\mathfrak{g}=11$, then $\mathfrak{g}$ is isomorphic to one of the nonisomorphic Lie algebras $\mathfrak{g}_{11}^{1}$

$$
\left\{\begin{array}{l}
{\left[X, Y_{1}\right]=Y_{1} ;\left[X, Y_{i}\right]=i Y_{i}, i=2, \cdots, 9 ;\left[X, Y_{10}\right]=11 Y_{10}} \\
{\left[Y_{1}, Y_{i}\right]=Y_{i+1}, i=2, \cdots, 8 ;\left[Y_{i}, Y_{11-i}\right]=(-1)^{i} Y_{10}, i=2, \cdots, 5} \\
{\left[Y_{2}, Y_{i}\right]=Y_{i+2}, i=3,4 ;\left[Y_{2}, Y_{i}\right]=-Y_{i+2}, i=6,7} \\
{\left[Y_{3}, Y_{i}\right]=Y_{i+3}, i=4,5 ;\left[Y_{4}, Y_{5}\right]=Y_{9} .}
\end{array}\right.
$$

$\mathfrak{g}_{11}^{2}$

$$
\left\{\begin{array}{l}
{\left[X, Y_{1}\right]=Y_{1} ;\left[X, Y_{i}\right]=i Y_{i}, i=2, \cdots, 9 ;\left[X, Y_{10}\right]=11 Y_{10}} \\
{\left[Y_{1}, Y_{i}\right]=Y_{i+1}, i=2, \cdots, 8 ;\left[Y_{i}, Y_{11-i}\right]=(-1)^{i} Y_{10}, i=2, \cdots, 5} \\
{\left[Y_{2}, Y_{i}\right]=Y_{i+2}, i=3,4 ;\left[Y_{2}, Y_{5}\right]=2 Y_{7} ;\left[Y_{2}, Y_{6}\right]=3 Y_{8} ;\left[Y_{2}, Y_{7}\right]=7 Y_{9}} \\
{\left[Y_{3}, Y_{i}\right]=-Y_{i+3}, i=4,5 ;\left[Y_{3}, Y_{6}\right]=-4 Y_{9} ;\left[Y_{4}, Y_{5}\right]=3 Y_{9}}
\end{array}\right.
$$

and $\mathfrak{g}$ is rigid and rational.

3. If dim $\mathfrak{g}=13$, then $\mathfrak{g}$ is isomorphic to one of the nonisomorphic Lie algebras described in Theorem 3 , and $\mathfrak{g}$ is rigid nonrational real or nonreal.

4. If $\operatorname{dim} \mathfrak{g} \geq 15$, then $\mathfrak{g}$ is nonrigid.

Proof. The parameters $a_{i j}$ appear in the definition of $B_{n}^{2}$. These parameters satisfy

$$
a_{i j}=a_{i j+1}+a_{i+1 j}
$$

Each constant $a_{i j}$ can be linearly expressed from $\alpha_{1}=a_{23}, \alpha_{2}=a_{34}, \cdots, \alpha_{t}=$ $a_{t+1, t+2}$ with $t=[n-3 / 2]$.

Suppose $\operatorname{dim} \mathfrak{g}=11$. The Jacobi conditions related to the $\alpha_{i}, \quad i=1,2,3$, are reduced to

$$
\left\{\begin{array}{l}
2 \alpha_{1}-\alpha_{2}-\alpha_{3}=0 \\
-2 \alpha_{1} \alpha_{3}+3 \alpha_{2}^{2}-\alpha_{2} \alpha_{3}=0
\end{array}\right.
$$

The solutions are $\left(\alpha_{1}, \alpha_{2}, \alpha_{3}\right)=\alpha_{1}(1,1,1)$ or $\alpha_{1}(1,-1,3)$ for $\alpha_{1} \in \mathbb{C}$. We have 3 cases:

$$
\begin{gathered}
\alpha_{1}=0:(0,0,0), \\
\alpha_{1} \neq 0:(1,1,1) \text { or }(1,-1,3) .
\end{gathered}
$$

In the last two cases, we can suppose, using a change of basis, that $\alpha=1$ and the corresponding Lie algebras are rigid. But the Lie algebra corresponding to the trivial solution $(0,0,0)$ has a nontrivial deformation and it is not rigid. 
Suppose $\operatorname{dim} \mathfrak{g}=13$. The Jacobi conditions related to $\alpha_{i}, i=1,2,3,4$, are reduced to

$$
\left\{\begin{array}{l}
2 \alpha_{1}-3 \alpha_{2}+\alpha_{4}=0 \\
-2 \alpha_{1} \alpha_{3}+3 \alpha_{2}^{2}-\alpha_{2} \alpha_{3}=0 \\
-2 \alpha_{1} \alpha_{4}+\alpha_{2}\left(-4 \alpha_{3}-\alpha_{4}\right)+\alpha_{3}\left(6 \alpha_{3}-\alpha_{4}\right)=0
\end{array}\right.
$$

If $\alpha_{3}=0$, then $\alpha_{i}=0, \quad i=1,2,4$, and the corresponding Lie algebra is not rigid. If $\alpha_{3} \neq 0$, a change of basis permits us to consider $\alpha_{3}=1$. The previous system leads to the equation:

$$
-9 \alpha_{2}^{4}+18 \alpha_{2}^{3}-5 \alpha_{2}^{2}-8 \alpha_{2}+6=0
$$

with $\alpha_{4}=-3 \alpha_{2}^{2}+4 \alpha_{2}$ and $\alpha_{1}=\frac{3 \alpha_{2}^{2}-\alpha_{2}}{2}$ whose solutions are $\alpha_{2}=\frac{1+\sqrt{10}}{3}$ or $\alpha_{2}=$ $\frac{1-\sqrt{10}}{3}$, or $\alpha_{2}=\frac{2+i \sqrt{2}}{3}$, or $\alpha_{2}=\frac{2-i \sqrt{2}}{3}$. As every change of basis which preserves the eigenvalues of the external torus and the decomposition into eigenspaces is rational, we can conclude that the last algebras are not rational.

Suppose $\operatorname{dim} \mathfrak{g} \geq 15$. Consider the subspace of $\mathfrak{n}$ generated by $Y, Y_{1}, \cdots, Y_{n-1}$. This is a nilpotent Lie algebra of type $A_{n}$ with the same $k=2$ and the same parameters $\alpha_{i}$. These algebras are classified in [2]. The parameters $\alpha_{i}$ are entirely determined. If we write the Jacobi conditions related to the last vectors, we can see that these solutions concerning the $\alpha_{i}$ don't satisfy the associated linear system. Thus the only solution for the parameters $\alpha_{i}$ is $\alpha_{i}=0$. Such algebras are not rigid.

Now suppose $k=3$. The results in this case are analogous to those of the previous section, but there are no nonrational rigid algebras:

1. In dimension 9, there is only one isomorphic class of Lie algebras, and each representative is rigid and rational (it corresponds to the case $k=n-1$ ).

2. If $\operatorname{dim} \mathfrak{g}=11$, then $\mathfrak{g}$ is not rigid.

3. For the dimension $=13$ (resp. 15 or 17), there are, respectively, 2, 4 or 2 classes of Lie algebras which are rigid and rational.

4. If $\operatorname{dim}(\mathfrak{g}) \geq 19$, there is only one isomorphism class of such Lie algebras, and $\mathfrak{g}$ is rigid and rational.

The proof is a direct computation of the Jacobi conditions reduced to the parameters $\alpha_{1}, \cdots, \alpha_{t}$.

The last case we consider is $k=4$. For dimensions less than 15 , this corresponds to the special case studied at the beginning. Other dimensions are summarized in the following.

Proposition 3. If $\operatorname{dim} \mathfrak{g}=17$, then $\mathfrak{g}$ is isomorphic to one of the following Lie algebras corresponding to the solutions

$$
\begin{gathered}
\left(\alpha_{1}, \alpha_{2}, \alpha_{3}, \alpha_{4}, \alpha_{5}\right)=(2,0,1,2,3),\left(\frac{8+\sqrt{114}}{8}, 1,5 / 4,5 / 2, \frac{-7+\sqrt{114}}{4}\right), \\
\left(\frac{8-\sqrt{114}}{8}, 1,5 / 4,5 / 2, \frac{-7-\sqrt{114}}{4}\right)
\end{gathered}
$$

which correspond to rigid Lie algebras. Only the first Lie algebra is rational.

If dimg $\geq 18$, then the nilradical is isomorphic to $Q_{n}$. 
Proof. Suppose $\operatorname{dim} \mathfrak{g}=17$. The Jacobi conditions related to $\alpha_{i}, \quad i=1,2,3,4,5$, are reduced to the four equations:

$$
\left\{\begin{array}{l}
2 \alpha_{1}-5 \alpha_{2}-\alpha_{3}+\alpha_{5}=0 \\
\alpha_{4}-2 \alpha_{3}=0 \\
-5 \alpha_{2}^{2}+6 \alpha_{2} \alpha_{3}-2 \alpha_{2} \alpha_{4}+4 \alpha_{1} \alpha_{3}-2 \alpha_{1} \alpha_{4}=0 \\
-5 \alpha_{2}^{2}+12 \alpha_{2} \alpha_{3}+2 \alpha_{2} \alpha_{4}-\alpha_{2} \alpha_{5}+4 \alpha_{1} \alpha_{3}-15 \alpha_{3}^{2}+10 \alpha_{3} \alpha_{4} \\
-\alpha_{3} \alpha_{5}+6 \alpha_{1} \alpha_{4}+2 \alpha_{1} \alpha_{5}=0
\end{array}\right.
$$

If $\alpha_{2} \neq 0$, we can take $\alpha_{2}=1$ and the solutions correspond to the second and third solutions of the proposition. If $\alpha_{2}=0$, then $\alpha_{3} \neq 0$, if not $\alpha_{i}=0$ for $i=1,2,3,4,5$ and the nilradical is $Q_{16}$ and this case is nonrigid. Take $\alpha_{3}=1$, then $\alpha_{4}=2, \alpha_{1}=\alpha_{5}-1$ and $\left(\alpha_{5}-3\right)^{2}=0$. This gives the first solution.

Conclusion. This study shows that it is not surprising to meet nonrational rigid Lie algebras because the conditions of Jacobi give a system of polynomial equations with integer coefficients for which the rigid Lie algebras correspond to isolated solutions. These solutions can be noninteger.

\section{REFERENCES}

1. Ancochea Bermudez, J.M., On the Rigidity of Solvable Lie Algebras. In, Deformation Theory of Algebras and Structures and Applications., Kluwer Academy Publisher, 1988, pp. 403-445. MR 90b:17019

2. Ancochea Bermudez, J.M., Goze, M., Le rang du système linéaire des racines d'une algèbre de lie résoluble rigide, Communications in Algebra 20 (1992), 875-887. MR 93a:17006

3. Ancochea Bermudez, J.M., Goze, M., Algèbres de Lie rigides dont le nilradical est filiforme, C.R.A.Sc.Paris 312 (1991), 21-24. MR 91m:17010

4. Carles, R., Variété d'algèbres de Lie, Thèse Poitiers 1984.

5. Goze, M., Khakimdjanov, Y., Nilpotent Lie Algebras, Math. Appl. 361, Kluwers editeur., 1996. MR 97e: 17017

6. Goze, M., Hakimjanov, Y., Sur les algèbres de Lie nilpotentes admettant un tore de dérivations, Manuscripta Math. 84 (1994), 115-224. MR 95g:17011

7. Goze, M., Perturbations of Lie Algebras. In, Deformation Theory of Algebras and Structures and Applications., Kluwer Academy Publisher, 1988, pp. 403-445. MR 90e: 17008

8. Sund, T., Classification of filiform solvable Lie algebras, Communications in Algebra 22:11 (1994), 4303-4359. MR 95e:17010

Universidad Complutense, Facultad de Matematicas, Departamento de geometria y TOPOLOGIA 27000 MADRID, SPAIN

Université de Haute Alsace, Faculté des Sciences et Techniques, 32, rue du GrillenBreit, F, 68000 Colmar, France

E-mail address: M.Goze@univ-mulhouse.fr 